\title{
A search for shock-excited optical emission from the outflows of massive young stellar objects ${ }^{\star}$
}

\author{
C. Alvarez ${ }^{1,2,3}$ and M. G. Hoare ${ }^{1}$ \\ 1 School of Physics and Astronomy, University of Leeds, Leeds LS2 9JT, UK \\ e-mail: alvarez@mpia-hd.mpg.de \\ 2 Kapteyn Astronomical Institute, Postbus 800, 9700 AV Groningen, The Netherlands \\ 3 Max-Planck-Institut für Astronomie, Königstuhl 17, 69117 Heidelberg, Germany
}

Received 10 August 2004 / Accepted 28 May 2005

\begin{abstract}
We have searched for optical shock-excited emission lines in the outer parts of the bipolar outflows from massive young stellar objects where the flow terminates and the extinction is expected to be low. The Taurus Tunable Filter (TTF) at the Anglo-Australian Telescope (AAT) was used to obtain narrow-band (12-15 ̊) images of the H $\alpha$, [NII] 6583, and [SII] 6717/6731 lines around Mon R2 IRS3, S255 IRS1/3, GL 961 and GL 989. No clear examples of shocked emission were found. A bow shock feature in the GL 989 region maybe related with the molecular outflow NGC 2264 D. A weak optical bow-like feature is seen in GL 961, but which of the stars in the cluster is the driving source cannot be uniquely identified. Other emission line features were found in Mon R2 and S255 that are consistent with an HII region origin. The observed fluxes and the upper limits were compared with predictions from radiative shock models available in the literature. Any head-on collisions of a jet with speeds of the order of $500 \mathrm{~km} \mathrm{~s}^{-1}$ should have been detected if the extinction $A_{\mathrm{v}}<10$ mag. Estimates of the extinction in the outer parts of the molecular clouds are somewhat lower than this, although it is possible that it could be higher local to any interaction regions due to swept up material. If the extinction is low, then the shocks must either be slower $\left(<150 \mathrm{~km} \mathrm{~s}^{-1}\right)$ or very oblique $\left(>75^{\circ}\right)$, which would argue against the presence of highly collimated jets from high mass young stellar objects.
\end{abstract}

Key words. stars: formation - ISM: jets and outflows

\section{Introduction}

Bipolar outflows appear to be a ubiquitous phenomenon during the formation of stars in all mass ranges (Bally \& Lada 1983; Henning et al. 2000; Ridge \& Moore 2001; Beuther et al. 2002). Low mass young stellar objects (YSOs) show highly collimated bipolar jets from a few 10 AU (Burrows et al. 1996) to several parsec (Reipurth et al. 1997; Eislöffel 2000). These jets are thought to be magneto-hydrodynamically driven by a star-disc system (e.g. X-wind, Shu et al. 1994). Alternatively, some models predict that they can be hydrodynamically driven (e.g. Konigl 1982; Delamarter et al. 2000). These jets can transfer momentum to the surrounding material forming the large scale molecular outflows (Masson \& Chernin 1994), although not all flows are necessarily jet-driven (Lee et al. 2001). They have also been shown to be a means of significant angular momentum transfer from the disc (Coffey et al. 2004).

Jets appear to be less common in massive star formation (Mundt \& Ray 1994). There are only a few cases where optical shock-excited emission has been detected close to the exciting source. Jet-like Herbig-Haro (HH) flows have been found in

^ Figures 6 to 14 are only available in electronic form at http://www . edpsciences.org
GL 4029 IRS1 (Ray et al. 1990) and GL 2591 and MWC 1080 (Poetzel et al. 1992). Their length varies between $10^{\prime \prime}$ and $30^{\prime \prime}$ (i.e. $0.15-0.5 \mathrm{pc}$ ) and they show speeds of up to $500 \mathrm{~km} \mathrm{~s}^{-1}$. This may, in part, be due to the fact that massive YSOs are generally more embedded than solar type young stars. However, searches for shock-excited $\mathrm{H}_{2}$ in the near-IR, where the extinction is low, have also found little evidence for jets. In the instances where jets are found, they are generally thought to be driven by low mass young stars located in the associated cluster (Davis et al. 1998; Wang et al. 2003). Kumar et al. (2002) again found a confused picture and appealed to the presence of dense clusters in massive star forming regions as a possible distorting mechanism. The lack of $\mathrm{H}_{2}$ emission associated with jets may indicate an actual lack of jets in massive YSOs. However, the presence of jets cannot be completely ruled out, since any jets from massive YSOs may be too fast for the $\mathrm{H}_{2}$ molecule to survive the shock. Evidence for fast winds in massive YSOs are inferred from the broad CO absorption lines seen in the near-IR (Mitchell et al. 1991).

Evidence for outflowing gas in massive YSOs is also found at scales of a few $100 \mathrm{AU}$ from the driving source in the form of an ionised wind. At these scales, the presence of an ionised wind is normally indicated by a radio continuum 
spectrum with a spectral index of $\sim 0.6$ (e.g. Tofani et al. 1995) and broad near-IR emission line profiles (Bunn et al. 1995). In some cases, the free-free radio emission from the inner wind shows a jet morphology (e.g. HH 80-81, Marti et al. 1993; Cep A, Torrelles et al. 1996; G35.2N, Gibb et al. 2003). Proper motion studies show that these jets are moving with speeds of about $500 \mathrm{~km} \mathrm{~s}^{-1}$ (Marti et al. 1998; Rodríguez et al. 2001). In other cases, the ionised wind appears to be rather equatorial (e.g. S140 IRS1, Hoare et al. 1996; Hoare 2002).

Theoretical models show that radiation pressure in massive young stars can drive gas off the surface of a disk, producing a predominantly equatorial wind (Proga et al. 1998; Drew \& Proga 2000). The relationship between these ionised winds and the large scale bipolar molecular flows is not known. Equatorial or wide-angled winds may only interact with the surrounding medium via very oblique shocks. The lower shock speeds may also then make the detection of optical lines difficult and would not result in the typical bow-shock morphology of jet interactions.

If jets are driven by massive YSOs then one might expect to find evidence of their interaction at some considerable distance from the exciting source. The CO flows are often very extended and the problem of high extinction should decrease as the blueshifted lobe emerges on the near-side of the molecular cloud. In the case of G192.16-3.82, several bow-shocks, but no jet, are seen towards the blue-shifted outflow lobe at distances $>2$ pc from the driving source (Shepherd et al. 1998). Cep A also has a complex of optical and molecular hydrogen emission (see Mundt \& Ray 1994; Goetz et al. 1998), although it is not aligned to the small scale bipolar flow from the main powering source.

In this work, we search for shock-excited optical emission (H $\alpha,[\mathrm{NII}]$ 6583, [SII] 6717/6731 lines) in the outflows in four well-studied massive YSOs (Mon R2 IRS3, S255 IRS1/3, GL 961 and GL 989). We study the outer parts of the outflow, at typical distances of a few parsecs from the driving source, where the extinction should be low. It is also at these distances where the $\mathrm{CO}$ outflows appear to end, although this will depend on the resolution and sensitivity of the observations especially if the flow breaks out of the cloud completely. If the flow is contained within the cloud, then it is here where a strong interaction of the flow with the interstellar medium (ISM) is expected and produce the optical line emission. Since massive star forming regions are typically located at distances $\gtrsim 1 \mathrm{kpc}$ from Earth, the shock-excited emission is expected to appear at several arcminutes from the driving source. Wide-field imaging is therefore needed to trace any optical shocks at the end of the $\mathrm{CO}$ outflow.

The observations presented here cover fields of view around the sources about five times larger than previous studies. Our results are compared with outflow indicators at other wavelengths (CO outflow morphology, near-IR shock-excited emission, near-IR sub-arcsecond morphology) to gain insight into the nature of the detected emission features. Further information about the nature of the emission is achieved by comparing the observed emission-line ratios and upper limits with predictions from radiative shock models found in the literature (Dopita \& Sutherland 1996).
Table 1. Log of observations.

\begin{tabular}{lcc}
\hline \hline Object Name & Orientation & Wavelength setting \\
\hline Mon R2 IRS3 N* & 0 & $\mathrm{H} \alpha$ \\
Mon R2 IRS3 N & 0 & {$[\mathrm{SII}]$} \\
Mon R2 IRS3 S * & 0 & $\mathrm{H} \alpha$ \\
Mon R2 IRS3 S & 0 & {$[\mathrm{SII}]$} \\
S255 IRS1/3 & 60 & $\mathrm{H} \alpha$ \\
S255 IRS1/3 & 60 & $\mathrm{H} \alpha$ \\
GL 961 NW & 135 & $\mathrm{H} \alpha$ \\
GL 961 NW & 135 & {$[\mathrm{SII}]$} \\
GL 961 SE** & 135 & {$[\mathrm{SII}]$} \\
GL 989 & 135 & $\mathrm{H} \alpha$ \\
GL 989 & 135 & {$[\mathrm{SII}]$} \\
\hline * Data taken through cirrus. & \\
** Seeing of 3".3. &
\end{tabular}

The observations and data reduction are described in Sect. 2. The main optical line-emission features detected in our images are discussed in Sect. 3. In Sect. 4, we compare the line ratios for the observed features and the upper limits for the non-detections with predictions from shock models. In Sect. 5, we briefly discuss the presence of $\mathrm{H} \alpha$ emission line stars in our images. The conclusions are presented in Sect. 6.

\section{Observations and data reduction}

\subsection{Optical observations}

Narrow-band imaging was performed on the massive YSOs listed in Table 1 using the Taurus Tunable Filter (TTF) at the Anglo Australian Telescope (AAT) on Nov. 23 and 24, 1999. Narrow band imaging with the TTF (Bland-Hawthorn \& Jones 1998), which is a Fabry-Perot interferometer, has the advantage that it provides a narrower band pass $(\Delta \lambda=12-15 \AA)$ compared with standard narrow band filters $(\Delta \lambda=50-100 \AA)$. Therefore, it was possible to separate the $\mathrm{H} \alpha$, [NII] $6583 \AA$ and [SII] 6717 and $6731 \AA$ lines as well as to cut down the sky and continuum background. The TTF also has the capability of charge shuffling synchronised with wavelength switching, which provides a better continuum subtraction than standard narrow-band imaging because the seeing changes between line and continuum images are minimised.

The images presented in this work were taken in the 3-shuffling observing mode, which allows the observation of two emission lines and a continuum band in rapid succession. Two instrumental settings were selected to detect typical emission lines from $\mathrm{HH}$ objects. One of them (the $\mathrm{H} \alpha$ setting) was chosen to image $\mathrm{H} \alpha$ and the [NII] $6583 \AA$ line, with the transmission for the continuum centred at $6637 \AA$. The other setting (the [SII] setting) was chosen to observe the [SII] 6717 and $6731 \AA$ lines, with the transmission for the continuum centred at $6673 \AA$. Two shuffle cycles of one minute per wavelength were used to generate a single dither exposure for the science targets. For a given instrumental setting, each single exposure contains (on three different areas of the CCD) all three images of the target (each corresponding with one of the three 
band-passes). Since 5 dither positions were used for each target, the total integration time on each line was $10 \mathrm{~min}$.

The detector used was MITLL2, which has $4 \mathrm{~K} \times 2 \mathrm{~K}$ pixels, and at $\mathrm{f} / 8$, yields a pixel scale of $0.37 \mathrm{pix}^{-1}$. The instrumental set-up yielded a FOV of $10^{\prime} \times 5^{\prime}$. The CCD was oriented with the large dimension of the FOV along the outflow direction. Two spatial positions were selected to cover $10^{\prime}$ on either side of the outflow (see Table 1). The seeing varied between 1".5 and 3". 3 during the whole observing period.

Before and after the observation of each science target, a pencil-beam scan of a neon lamp was taken, which was used for wavelength calibration. The instrument was stable for most of the night, showing drifts of $\sim 1 \AA$. Drifts of 6-10 $\AA$ occurred at the beginning and the end of each night, which are thought to be caused by temperature variations.

The TTF has a phase shift of $\sim 20 \AA$ from the centre to the edge of the field of view (FOV). Due to this phase shift, the peak transmission at the centre was chosen at $+8 \AA$ with respect to the emission line of interest in an attempt to maximise the FOV sensitive to the line. Hence, our images have a maximum sensitivity to the line within a ring of radius $\sim 2^{\prime}-4^{\prime}$. The sensitivity drops by a factor of $\sim 4$ towards the centre of the FOV. The massive YSO was located in the high sensitivity ring. In the $\mathrm{H} \alpha$ setting, the maximum transmission (at the optical axis) was centred at $6571 \AA, 6591 \AA$ and $6637 \AA$. In the [SII] setting, the maximum transmission (at the optical axis) was centred at $6724 \AA, 6738 \AA$, and $6673 \AA$.

The spectrophotometric standard LTT 1020 (Gunn \& Stryker 1983) was used for the flux calibration of the images. The calibration constants were obtained by dividing the flux from the template spectrum of LTT 1020 (available at http: //www . eso.org/observing/standards/spectra/) by the number of counts resulting from aperture photometry within an aperture of radius $10^{\prime \prime}$. Although the quality of the photometry for the data from Gunn \& Stryker is $1 \%$, their low sampling $(\Delta \lambda=20 \AA)$ induces an error of about $5 \%$ in the flux from the template spectrum of LTT 1020 near $\mathrm{H} \alpha$. A further $2 \%$ error also contributed to the calibration due to temporal fluctuations on the etalon spacing and the lack of perfect plate parallelism. The presence of cirrus during Nov. 24, also contributes to the higher error for the calibration constants from this night. Therefore, the flux calibration from Nov. 23 was applied to the data from both nights.

Twilight and dome flatfields were taken at the beginning and end of each night. Twilight flatfields were taken without the TTF in the optical path. Dome flatfields were taken at each wavelength setting. An average dome flat was produced for each observed line. Each night, an average twilight flat was divided by each dome flat and was highly smoothed to reproduce large scale illumination gradients. Each dome flatfield was divided by this illumination correction to generate a sky corrected flatfield. Each on-source dither exposures at each wavelength setting was divided by its corresponding sky-corrected flatfield. The sky images for each wavelength setting were created by median average of several on-source exposures. The sky images were subtracted off each science frame.

Even though the TTF provides a very narrow band pass, the science frames for each emission line can be contaminated by adjacent lines due to the phase shift which varies radially from the optical axis. For instance, in the [SII] setting, the frame at $6724 \AA$, which was chosen to observe the [SII] 6717 line, is equally as sensitive to the [SII] 6717 as to the [SII] 6731 line at the optical axis. At $3^{\prime}$ from the optical axis, the sensitivity to [SII] 6717 rises up to $90 \%$, while the contamination by [SII] 6731 decreases below 5\%. Essentially, the light detected on each science frame is a linear combination of several emission lines and the continuum. It was, therefore, necessary to subtract the continuum and to invert the linear combination to disentangle the contribution from each emission line to the total flux.

The coefficients in the linear combination are basically the phase-shift calibration images, which represent the sensitivity of the Fabry-Perot on each pixel to a particular emission line. Three phase-shift calibration images were needed for the $\mathrm{H} \alpha$ setting, since there are three emission lines that could contribute in this spectral region (H $\alpha$, [NII] 6583 and $6548 \AA$ ). For the [SII] setting, only two phase-shift calibration frames were required, since only two lines ([SII] 6717 and $6731 \AA$ ) are included in this region of the spectrum. Phase-shift calibration exposures were taken using the Neon $6599 \AA$ line. However, due to the presence of drifts in the etalon spacing across the night, it was necessary to use modeled phase-shift calibration images suited for each target. It was found that a drift of $1 \AA$ generates a $12 \%$ discrepancy in the line fluxes, while a drift of $4 \AA$ produces a $25 \%$ discrepancy. Furthermore, the modeled calibration images did not reproduce exactly the observed images, due to the presence of some background emission in the Ne calibration lamp. This discrepancy induces a systematic error of $15 \%$ to the fluxes measured in the phase-shift-calibrated images. The [NII] line at $6548 \AA$ was assumed to be a factor of 3 fainter than [NII] 6583 to generate the final phase-shiftcalibrated images.

For a each target, the line images (i.e. continuum-subtracted and phase-shift calibrated images) were aligned and averaged to form mosaics. The final mosaics were cropped to a size of $4.5 \times 8^{\prime}$, since the noise rises sharply in the outer parts of the FOV, where the phase shift is not well reproduced by our phase-shift model. The mosaics where smoothed using a grid of $4 \times 4$ pixels to enhance the contrast in order to facilitate the identification of faint extended features.

A plate solution was fitted using stars that were identified in the DSS plates. This yields source positions with an accuracy better than 0.2 . Near-IR seeing-limited images were registered in the final TTF images to locate the YSO and near-IR nebulosity. For Mon R2 IRS3 and S255 IRS1, $K_{\mathrm{s}}$ images from the 2 MASS $^{1}$ survey were utilised. For GL 961 and GL 989, $K$ band images taken at UKIRT were used (see details below). The astrometry for the UKIRT images, was computed in two steps. Firstly, a plate solution was found for the wide field $K_{\mathrm{s}}$

\footnotetext{
1 This publication makes use of data products from the Two Micron All Sky Survey, which is a joint project of the University of Massachusetts and the Infrared Processing and Analysis Center/California Institute of Technology, funded by the National Aeronautics and Space Administration and the National Science Foundation.
} 
Table 2. Emission line features ${ }^{a}$.

\begin{tabular}{|c|c|c|c|c|c|c|c|c|}
\hline Region & ID & $\alpha(\mathrm{hms})$ & $\delta\left({ }^{\circ \prime \prime \prime}\right)$ & $\mathrm{H} \alpha$ & {$[\mathrm{NII}] 6583$} & [SII]6717 & [SII]6731 & Radius $^{b}$ \\
\hline \multirow[t]{3}{*}{ Mon $\mathrm{R} 2^{c, d}$} & A & 060747.0 & -062259 & $4.7 \pm 0.5$ & $1.2 \pm 0.1$ & $0.3 \pm 0.2$ & $0.4 \pm 0.2$ & 7.5 \\
\hline & B & 060747.2 & -062312 & $4 \pm 1$ & $1.0 \pm 0.2$ & $<0.6$ & $<0.6$ & 4 \\
\hline & $\mathrm{C}$ & 060745.6 & -062212 & $1.0 \pm 0.3$ & $<0.7$ & $<0.6$ & $<0.6$ & 7.5 \\
\hline S255 & A & 061255.1 & 175928 & $2.0 \pm 0.2$ & $<0.6$ & - & - & 5.5 \\
\hline \multirow[t]{2}{*}{ GL 961} & $\mathrm{~A}^{*}$ & 063435.7 & 041245 & $2.8 \pm 0.1$ & $0.3 \pm 0.1$ & $<0.3$ & $<0.3$ & 5.5 \\
\hline & $\mathrm{B}^{* *}$ & 063437.9 & 041313 & $1.0 \pm 0.2$ & $<0.3$ & $<0.3$ & $<0.3$ & 2 \\
\hline GL $989^{e}$ & A & 064110.0 & 092936 & $6.7 \pm 0.2$ & $<0.5$ & $<0.4$ & $<0.4$ & 7.5 \\
\hline
\end{tabular}

a The average surface brightnesses (or $3 \sigma$ upper limits) are quoted in the CGS system $\left(10^{-5} \mathrm{erg} \mathrm{s}^{-1} \mathrm{~cm}^{-2} \mathrm{sr}^{-1}\right.$ ) to facilitate the comparison with the models.

$b$ Radius in arcsecond used for the aperture photometry.

$c$ Absolute values should be taken with caution, since the data were taken through cirrus.

$d$ TTF drift of $7 \AA$ may introduce additional systematic errors in absolute calibration.

e TTF drift of $10 \AA$ Å may introduce additional systematic errors in absolute calibration.

* Emission star embedded in a nebulosity (GL 961-Fan).

** Emission star surrounded by a faint bow shock feature.

images from Hodapp (1994) using stars that are also seen in the DSS plates. Secondly, the plate solution was propagated to the UKIRT images. The accuracy achieved was $\$ 0$ ' 07 . In all cases, there were more than 10 identified stars to fit the plate solution.

\subsection{Near-IR observations}

On December 17, 1997, near-IR imaging of GL 961 and GL 989 was performed with IRCAM3 at UKIRT. The three broad-band near-IR filters $(J, H$ and $K)$ were used at a pixel scale of $0.286 \mathrm{pix}^{-1}$. A dithering technique was used and sky frames were taken interspaced with on-target observations. Flat-fields for each filter were produced by median average of sky exposures. Each image was sky-subtracted and divided by its corresponding flat-field. After fixing bad pixels, the images were aligned, and averaged to produce the final near-IR mosaics.

\section{Results}

In this section, the final mosaics are presented and the nature of the main optical emission line features detected in each massive star forming region is discussed. Here, we use the term "continuum-subtracted image" to refer to an image that was continuum-subtracted and phase-shift calibrated, i.e. an image that contains only pure line emission (see Sect. 2). We do not show all continuum-subtracted images in which no lineemission feature at all was detected within the field.

Table 2 shows the observed fluxes of the main optical emission features. For the sources where emission features are detected, the flux was computed using aperture photometry within the radius given in Col. 9. In these cases, an identifier and the position of the knot is also given. For the sources where no feature was detected, an estimate of the upper limit for the emission is given based on the $3 \sigma$ noise level. The noise is not uniform across the FOV, since the sensitivity varies spatially due to the phase shift. The typical $(1 \sigma)$ sensitivity of our final images is $\sim 3$ Rayleighs ( 1 Rayleigh $=3 \times 10^{-7} \mathrm{erg} \mathrm{s}^{-1} \mathrm{~cm}^{-2} \mathrm{sr}^{-1}$ ), which is slightly better than in some of the current and previous $\mathrm{H} \alpha$ surveys (see the web page of the new AAO/UKST H $\alpha$ survey $^{2}$ for a survey list). Where features are detected only in the $\mathrm{H} \alpha$ setting, the noise in the other settings is estimated at the same position. If no feature is found in either of the wavelength settings, the noise is estimated in the region of the FOV where the sensitivity is highest. The uncertainties quoted in Table 2 are the statistical errors. These errors are calculated from the dispersion in the fluxes obtained by aperture photometry of different radii. Therefore, the features in which the background is variable (e.g. due to the presence of a nearby extended feature) show higher errors. The systematic errors associated with the flux calibration ( $8 \%$ and $2 \%$ in the $\mathrm{H} \alpha$ and [SII] settings respectively), with the Ne lamp $(\sim 15 \%)$ and with the phase shift $(\sim 12-25 \%)$, should also be taken into account. The systematic errors do not affect the flux ratios between two lines from the same setting, which will be dealt with in Sect. 4.

\subsection{Mon R2 IRS3}

Mon R2 IRS3 is the brightest of a cluster of compact IR sources with associated extended emission located at a distance of 950 pc (Beckwith et al. 1976; Thronson et al. 1980). IRS3, with a luminosity of $6 \times 10^{3} L_{\odot}$, is located at approximately $30^{\prime \prime}$ to the east of a blister type HII region of 27" diameter (Massi et al. 1985). The HII region is powered by IRS1 SW, which is an embedded B0V star (Howard et al. 1994). The light scattered off the dust associated with the region forms a ring-shaped reflection nebula with a polarisation pattern centrosymmetric around IRS2, which is the main illuminating source (Aspin \& Walther 1990; Yao et al. 1997). The cluster is located near the centre of a giant molecular outflow (Bally \& Lada 1983; Wolf et al. 1990) oriented at a PA $\sim 135^{\circ}$ with the approaching gas flowing to the NW. However, the outflow morphology is

\footnotetext{
${ }^{2}$ http://www.ast.cam.ac.uk/AAO/local/www/qap/work/ table.jpg
} 

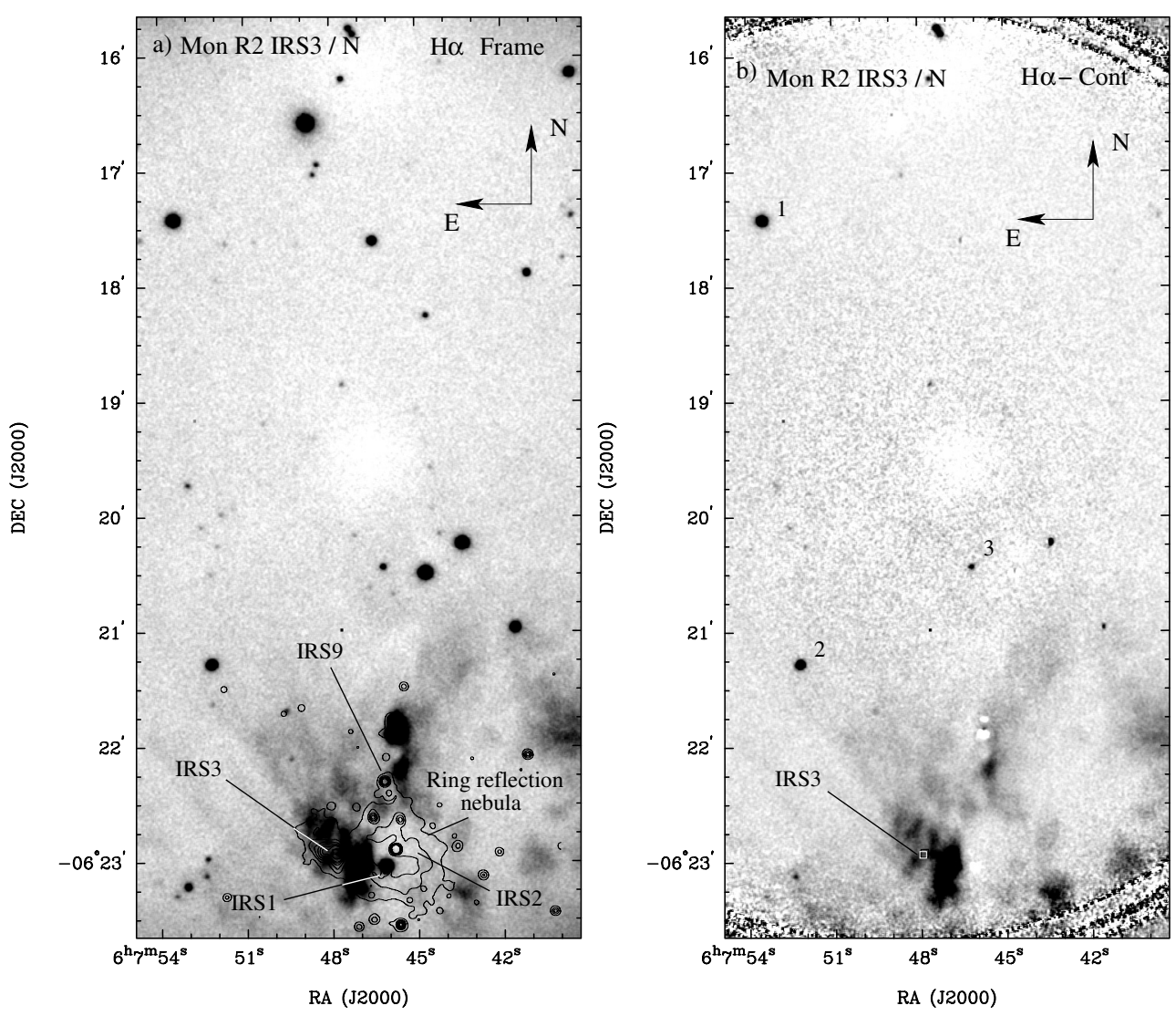

Fig. 1. a) Mosaic covering the northern region of Mon R2 in the $\mathrm{H} \alpha$ setting before continuum subtraction. The greyscale varies from $-3.1 \times 10^{-6}$ (light) to $3.4 \times 10^{-5} \mathrm{erg} \mathrm{s}^{-1} \mathrm{~cm}^{-2} \mathrm{sr}^{-1}$ (dark). The contours correspond to the $K_{\mathrm{s}}$ band image taken from 2MASS survey. The contours are at 10.0, $11.0,12.0,12.4,12.6,12.8$ and $13.0 \mathrm{mag} / \mathrm{arcsec}^{2}$. The labels indicate the major near-IR features. b) $\mathrm{H} \alpha$, continuum-subtracted image resulting from the mosaic shown in panel a). The greyscale varies between $-3.1 \times 10^{-6} \mathrm{erg} \mathrm{s}^{-1} \mathrm{~cm}^{-2} \mathrm{sr}^{-1}$ and $\quad 5.5 \times 10^{-5} \mathrm{erg} \mathrm{s}^{-1} \mathrm{~cm}^{-2} \mathrm{sr}^{-1}$. The white box at the bottom of the FOV indicates the position of Mon R2 IRS3. The original size of $5^{\prime} \times 10^{\prime}$ has been cropped to $4.5 \times 8^{\prime}$ since the noise rises sharply towards the edges of the FOV. Emission line stars in the field are labeled with the numbers 1 to 3 .

complex and it is not clear which of the sources is powering this outflow.

Speckle imaging reveals that IRS3 consists of two sources, IRS3 N (which is itself split into two stars) and S, a star with a prominent monopolar reflection nebula towards the S-SW, indicating outflow activity towards the observer at PA $\sim 198^{\circ}$ (Koresko et al. 1993; Preibisch et al. 2002; Alvarez et al. 2004). Therefore, two spatial settings were chosen, covering $10^{\prime}$ towards the north and $10^{\prime}$ towards the south of Mon R2 IRS3. The observations by Preibisch et al. (2002) also reveal a jet from IRS3 $\mathrm{N}$ at $\mathrm{PA} \sim 80^{\circ}$.

Figure 1a shows the $\mathrm{H} \alpha$ image (before continuum subtraction) in the northern spatial setting. The transmission for these lines is maximised at the position of the IR source. Several extended emission features are detected within $1^{\prime}$ of Mon R2 IRS3 that turn out to be pure line emission after the data are processed in order to subtract the continuum emission (see Fig. 1b). It can be seen that the line emission is stronger towards the SW of Mon R2 IRS3. Figure $1 \mathrm{~b}$ also illustrates the variation of the noise level across the FOV. Near the optical axis, the $3 \sigma$ level is at $3.7 \times 10^{-5} \mathrm{erg} \mathrm{s}^{-1} \mathrm{~cm}^{-2} \mathrm{sr}^{-1}$, it decreases to $1 \times 10^{-5} \mathrm{erg} \mathrm{s}^{-1} \mathrm{~cm}^{-2} \mathrm{sr}^{-1}$ near IRS3 and increases again to $>2.5 \times 10^{-4} \mathrm{erg} \mathrm{s}^{-1} \mathrm{~cm}^{-2} \mathrm{sr}^{-1}$ towards the edges of the FOV.

A faint cone of emission is seen extending to the north of IRS3 for about $2^{\prime}$ with an opening angle of about $60^{\circ}$. This may correspond to a lobe of redshifted $\mathrm{CO}$ of similar orientation and size that is seen in the CO map of Wolf et al. (1990). It is also possible that this is the large scale redshifted counterpart of the sub-arcsecond monopolar reflection nebula from IRS3 S. However, this faint cone of diffuse emission could also be due to a more general low density ionised halo in the region obscured by the dense molecular clumps surrounding Mon R2 IRS3 and the Mon R2 HII region.

Figure 2a shows the $\mathrm{H} \alpha$ image for a region that covers $7^{\prime}$ towards the south of Mon R2 IRS3. The continuum-subtracted $\mathrm{H} \alpha$ image of the same region (see Fig. 2b) shows the same emission features as in Fig. 1b, plus some very faint diffuse emission $\sim 1^{\prime} \mathrm{SW}$ of the HII region. Most of the field stars that appear in Figs. 1a and $2 \mathrm{a}$ are not present in the continuumsubtracted images. This is the result of the good continuum subtraction provided by the TTF due to charge shuffling. However, there are a few stars that appear to emit in $\mathrm{H} \alpha$. These are discussed in more detail in Sect. 5.

A close-up view of the optical line emission near IRS3 shows two knots, one to the $\mathrm{W}$ (knot $\mathrm{A}$ ) and the other one SW (knot B) of IRS3 (see Fig. 3). A third knot (C), fainter than the previous two, is located at about $30^{\prime \prime}$ to the NW of IRS3. There is also some patchy extended emission within about $20^{\prime \prime}$ of IRS3, which lies slightly above the noise level $\left(3 \sigma \sim 1 \times 10^{-5} \mathrm{erg} \mathrm{s}^{-1} \mathrm{~cm}^{-2} \mathrm{sr}^{-1}\right)$ in the $\mathrm{H} \alpha$ image. The brightest knot (A) is detected in [NII] 6583 (see Fig. 4), while knots B and $\mathrm{C}$ are only barely detected above the noise.

The location of knot A suggests that this feature may be associated with the HII region excited by IRS1 (Massi et al. 1985) rather than being of a shock-excited nature. The $5 \mathrm{GHz}$ map of the HII region shown in Massi et al. (1985) indicates that the radio flux density at the position of knot $\mathrm{A}$ is $\sim 6.5 \mathrm{mJy} \operatorname{arcsec}^{-2}$. If the radio emission is optically thin and the gas has a temperature of $\sim 10^{4} \mathrm{~K}$, a H $\alpha$ flux of $\sim 0.28 \mathrm{erg} \mathrm{s}^{-1} \mathrm{~cm}^{-2} \mathrm{sr}^{-1}$ is predicted from recombination theory for an HII region (e.g. 

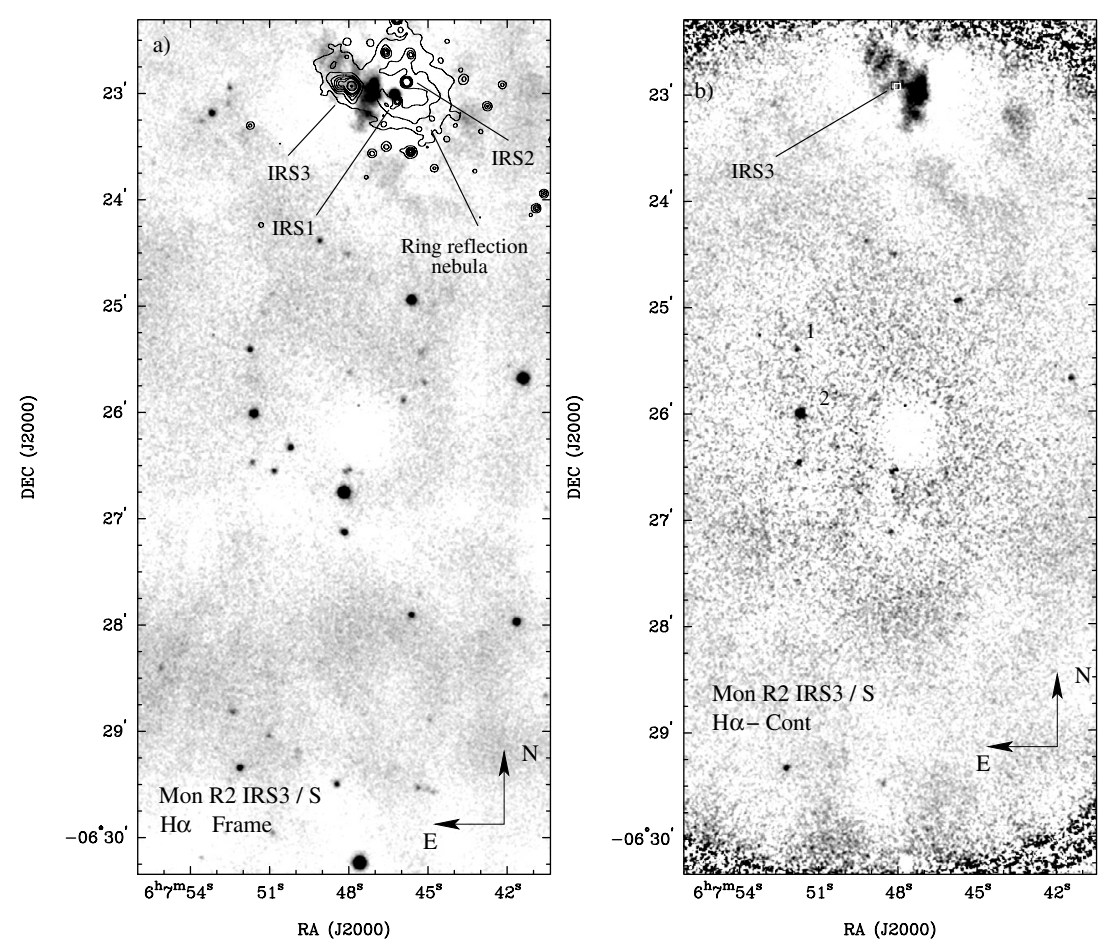

Fig. 2. a) Mosaic covering the southern region of Mon R2 in the $\mathrm{H} \alpha$ setting before continuum subtraction. The greyscale varies from $-3.1 \times 10^{-6} \mathrm{erg} \mathrm{s}^{-1} \mathrm{~cm}^{-2} \mathrm{sr}^{-1} \quad$ (light) to $3.4 \times 10^{-5} \mathrm{erg} \mathrm{s}^{-1} \mathrm{~cm}^{-2} \mathrm{sr}^{-1}$ (dark). The contours correspond to the $K_{\mathrm{s}}$ band image taken from the 2MASS survey. The labels indicate the major near-IR features. b) Continuumsubtracted $\mathrm{H} \alpha$ image resulting from the mosaic shown in panel a). The greyscale varies between $-3.1 \times 10^{-6} \mathrm{erg} \mathrm{s}^{-1} \mathrm{~cm}^{-2} \mathrm{sr}^{-1}$ and $3.4 \times 10^{-5} \mathrm{erg} \mathrm{s}^{-1} \mathrm{~cm}^{-2} \mathrm{sr}^{-1}$. The white box at the top of the FOV indicates the position of Mon R2 IRS3. Emission line stars are numbered with numbers 1 and 2 .

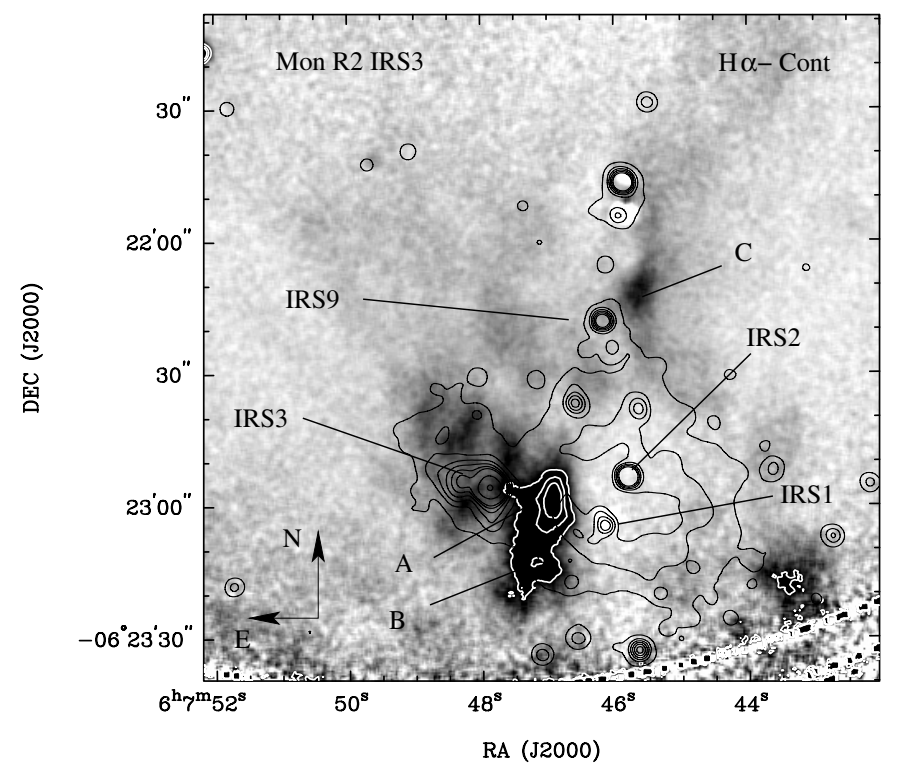

Fig. 3. $\mathrm{H} \alpha$ emission near Mon R2 IRS3. The greyscale is the same as in Fig. 1b. The thick white contours trace the $\mathrm{H} \alpha$ emission at 0.6, 1.1, and $1.6 \times 10^{-4} \mathrm{erg} \mathrm{s}^{-1} \mathrm{~cm}^{-2} \mathrm{sr}^{-1}$. The black contours trace the near-IR emission. The brightest optical emission features are labeled A, B and C. The rest of the labels indicate the brightest near-IR sources.

Osterbrock 1989) in the absence of extinction. The ratio between the predicted flux and the observed flux yields a visual extinction of $\sim 13 \mathrm{mag}$ at the location of knot A. This location appears to correspond with a region of low obscuration in the extinction map shown by Howard et al. (1994). Our extinction value is in good agreement with the estimate given by Howard et al. (1994). Therefore, the emission from knot A is consistent with being part of the HII region excited by IRS 1 .

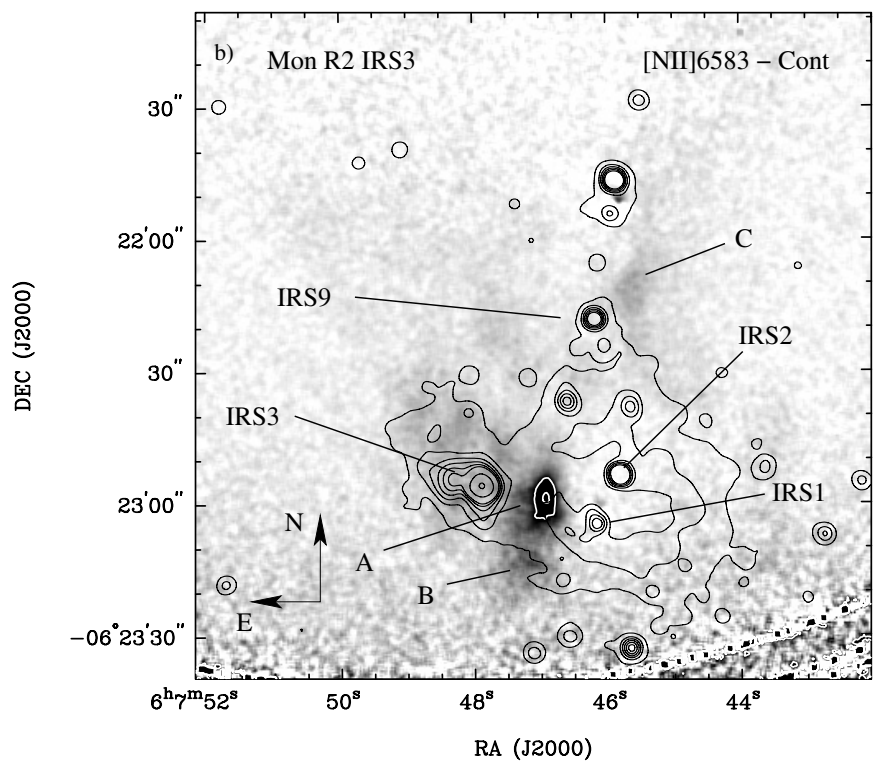

Fig. 4. [NII] 6583 emission near Mon R2 IRS3. The greyscale is the same as in Fig. 1b. The thick white contours trace the [NII] 6583 emission at $0.6,1.1$, and $1.6 \times 10^{-4} \mathrm{erg} \mathrm{s}^{-1} \mathrm{~cm}^{-2} \mathrm{sr}^{-1}$. The black contours trace the near-IR emission. The same optical knots as in Fig. 3 are labeled. For identification of the near-IR sources, see Fig. 3.

Knot B, however, is not located within the HII region. It is oriented at $\mathrm{PA} \sim 205^{\circ}$ with respect to IRS3. This value is in good agreement with the orientation of the blueshifted outflow lobe inferred from the near-IR speckle images (PA $\sim 198^{\circ}$, Alvarez et al. 2004). Note that it is precisely towards the blueshifted outflow lobe (i.e. the near side of the cloud) where any shock-excited optical emission is expected to be seen, although its morphology is rather diffuse and not reminiscent of shock interaction. Knot $\mathrm{C}$ is at $\mathrm{PA} \sim 320^{\circ}$ with respect to IRS3, 


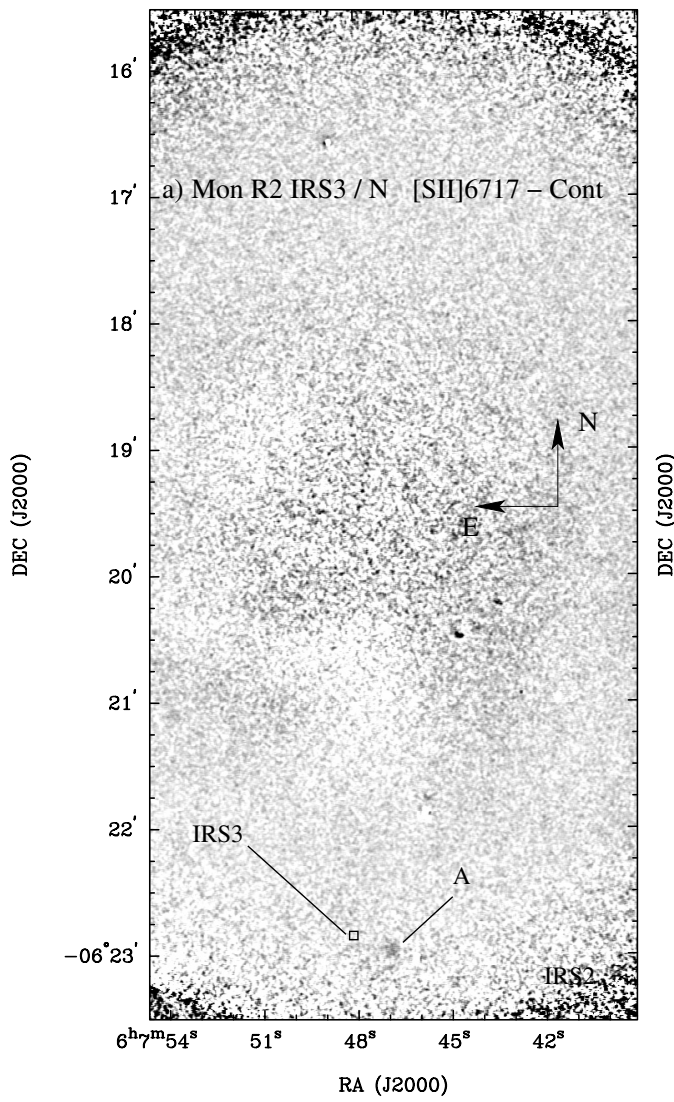

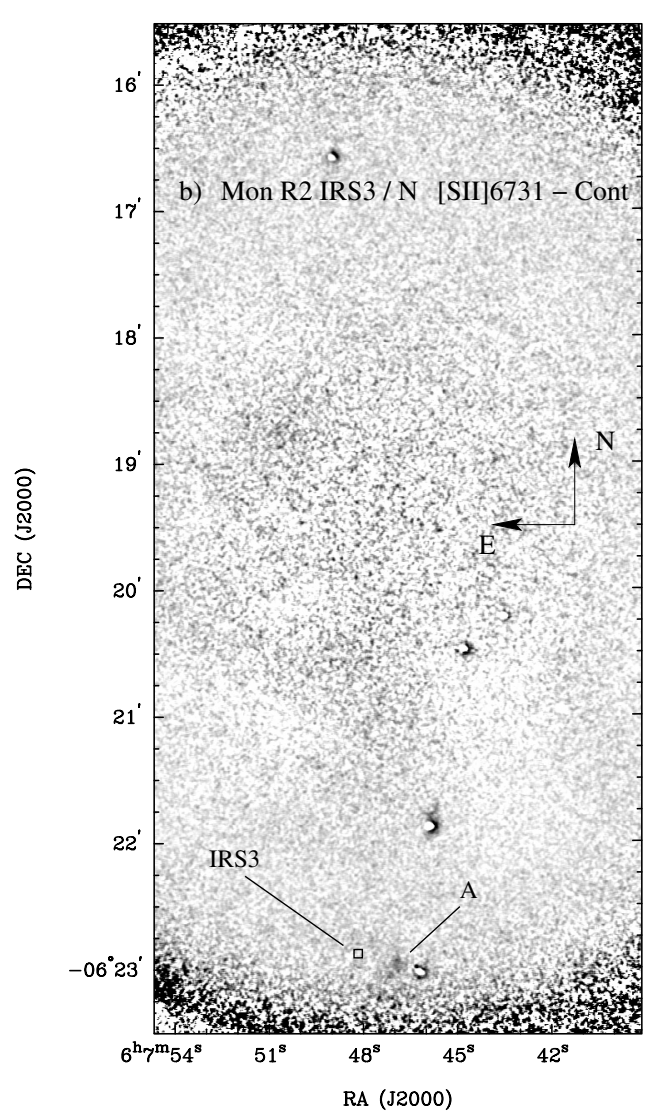

Fig. 5. a) Continuum-subtracted [SII]6717 image of the northern area in Mon R2. The greyscale varies from $-3.1 \times 10^{-6} \mathrm{erg} \mathrm{s}^{-1} \mathrm{~cm}^{-2} \mathrm{sr}^{-1}$ (light) to $\quad 3.1 \times 10^{-5} \mathrm{erg} \mathrm{s}^{-1} \mathrm{~cm}^{-2} \mathrm{sr}^{-1}$ (dark). b) Continuum-subtracted [SII]6731 image. The greyscale is the same as in panel a). The $3 \sigma$ level is $0.6 \times 10^{-5} \mathrm{erg} \mathrm{s}^{-1} \mathrm{~cm}^{-2} \mathrm{sr}^{-1}$ near the IR source and $1.8 \times 10^{-4} \mathrm{erg} \mathrm{s}^{-1} \mathrm{~cm}^{-2} \mathrm{sr}^{-1}$ near the centre of the FOV. The black square at the bottom of the FOV indicates the position of Mon R2 IRS3. which indicates that it is probably not related with the IRS3 outflow.

The northern and southern regions of the Mon R2 star forming region were also observed in the [SII] setting. Here we show only the images corresponding to the northern spatial setting, since no line emission features are seen in the southern setting. Figure 5a shows the northern [SII] frame (before continuum subtraction) which corresponds with a maximum transmission wavelength at the optical axis of $6724 \AA$. The [SII]6731 line image (continuum-subtracted) of the same FOV is shown in Fig. 5b. The feature A is marginally detected in both [SII] continuum subtracted images (see Table 2). From the flux ratio $[\mathrm{SII}] 6717 /[\mathrm{SII}] 6731=0.7 \pm 0.6$ it is not possible to give any constraints in the density.

\section{2. $S 255 \operatorname{IRS} 1 / 3$}

S255 IRS1 and IRS3, with a luminosity of $6.4 \times 10^{4} L_{\odot}$, form part of a cluster of IR sources located between the evolved HII regions S255 and S257 at a distance of $2.5 \mathrm{kpc}$ (Georgelin et al. 1973; Evans et al. 1977). A molecular outflow along the $\mathrm{N}-\mathrm{S}$ direction with its approaching lobe towards the south appears to be centred near the IR cluster (Heyer et al. 1989) and a second possible flow can be seen at a PA of about $60^{\circ}$ with blueshifted lobe to the SW. Infrared polarisation images by Tamura et al. (1991) show a bipolar nebula (IRN1) at PA 63, which indicates the presence of an outflow in the same direction. The location and orientation of the polarisation disc points at IRS $3^{3}$, located $\sim 2^{\prime \prime} \mathrm{E}$ of IRS1, as the outflow driving source. Sub-arcsecond resolution imaging of the region shows that the southern lobe of IRN1 is a conical nebula with IRS3 at its apex (Hoare et al. 1996; Alvarez et al. 2004). Howard et al. (1997) detect $\mathrm{Br} \gamma$ emission that appears to be elongated along the same direction as the bipolar nebula IRN1. Miralles et al. (1997) find molecular hydrogen emission from $\mathrm{HH}$-like objects along the presumed jet direction.

The S 255 IR region was observed with the TTF in the $\mathrm{H} \alpha$ setting at two spatial positions with the largest dimension of the FOV along the presumed jet direction. Figure ??a shows the $\mathrm{H} \alpha$ frame towards the SW jet lobe. After continuum subtraction, line emission that can be related with the IR cluster is found only at the position of IRS2 (Fig. ??b). No emission along the outflow direction was found, in particular where the $\mathrm{CO}$ outflow terminates. Although the transmission for the $\mathrm{H} \alpha$ and [NII] lines is lower near the centre of the FOV, the evolved HII regions S255, S256 and S257 are bright enough to be detected. The $3 \sigma$ noise is $1 \times 10^{-5} \mathrm{erg} \mathrm{s}^{-1} \mathrm{~cm}^{-2} \mathrm{sr}^{-1}$ near the IR cluster and it is 2.5 times larger near S257. The region to the NE of the YSO (see Fig. ??) does not show any line emission feature that can be associated with the outflow.

The continuum-subtracted $\mathrm{H} \alpha$ image (Fig. ??b) shows that several of the brighter field stars appear as negative features. We believe that several of these are genuine detections of stellar $\mathrm{H} \alpha$ absorption lines - see Sect. 5 .

\footnotetext{
${ }^{3}$ Here, we adopt the nomenclature "IRS\#" with numbers from Tamura et al. (1991) (e.g. our IRS3 corresponds with NIRS3 in Tamura et al. 1991).
} 
IRS2 coincides with the position of the compact HII region S255-2b seen by Snell \& Bally (1986). They estimated an integrated flux density at $5 \mathrm{GHz}$ of $3.2 \times 10^{-3} \mathrm{Jy}$. This value yields an $\mathrm{H} \alpha$ flux of $3.2 \times 10^{-12} \mathrm{erg}, \mathrm{s}^{-1} \mathrm{~cm}^{-2}$, which results from hydrogen recombination theory in an HII region (Osterbrock 1989). The observed flux integrated over a $7{ }^{\prime \prime} 5$ radius aperture (see Table 2) is $8.3 \pm 0.8 \times 10^{-14} \mathrm{erg} \mathrm{s}^{-1} \mathrm{~cm}^{-2}$. Therefore, an extinction $A_{\mathrm{v}} \sim 5 \mathrm{mag}$ is estimated towards feature $\mathrm{A}$ if it is produced in the HII region associated with IRS2 (the reddening law of $\mathrm{He}$ et al. 1995 has been assumed). This feature is located near the HH-like object $\mathrm{H} 28$ and counter-jet found by Miralles et al. (1997). However, the optical emission is most likely formed in the HII region.

\section{3. $G L 961$}

GL 961 is a luminous $\left(7.8 \times 10^{3} L_{\odot}\right)$ young stellar object that is located in the south-west rim of the Rosette nebula at a distance of $1.6 \mathrm{kpc}$ (Castelaz et al. 1985). This source is very faint in the optical (Eiroa 1981; Beetz et al. 1976), and lies approximately $30^{\prime \prime} \mathrm{E}$ of an optical fan-shaped nebula (GL 961-Fan). Lenzen et al. (1984) found that GL 961 is actually composed of two close IR objects, separated by about $5^{\prime \prime}$ (8000 AU) at a PA $\sim 70^{\circ}$. Castelaz et al. (1985) show that the spectral energy distribution of GL 961-E dominates at wavelengths longer than $2.2 \mu \mathrm{m}$, while the western object is brighter at shorter wavelengths.

GL 961 shows a complex outflow morphology in the nearIR (see Aspin 1998). Two main ejection events, at PA $\sim 0^{\circ}$ and $\mathrm{PA} \sim 40^{\circ}$ can be inferred from the bow shock emission features seen in $\mathrm{H}_{2}$ by Aspin. The origin of these ejection events is not clear, although the bow shocks appear to converge towards GL 961-E/W. The double source is located at the centre of a $4^{\prime}$ long molecular outflow oriented in the SE-NW direction with the approaching lobe towards the NW (Lada \& Gautier 1982).

This region was observed with TTF at two spatial positions, one towards the NW and the other to the SE of the IR sources GL 961-E and GL 961-W. Figure ?? shows a mosaic of the $\mathrm{NW}$ region in the $\mathrm{H} \alpha$ setting. After continuum subtraction, two emission features are detected in the $\mathrm{H} \alpha$ line (labeled $\mathrm{A}$ and $\mathrm{B}$ in Fig. ??). In the continuum-subtracted image [NII] $6583 \mathrm{im}$ age (see Fig. ??) only A is above the noise. No features are seen where the $\mathrm{CO}$ flow terminates some $2^{\prime}$ from the exciting source.

The optical line emission near the IR sources is shown in detail on figures ?? and ??. The brightest feature, A, is the extended nebula GL 961-Fan (Eiroa 1981). Its $\mathrm{H} \alpha$ emission is characterised by a central core with an extended halo towards the NW and SE. Figure ?? also shows GL 961-Fan in the [NII] 6583 transition. Our near-IR image and previous speckle imaging of GL 961-Fan (Alvarez et al. 2004) indicate that feature $\mathrm{A}$ is a star in nebulosity. It is likely that the nebula is being excited by the star. Feature B has a bow shock morphology with an $\mathrm{H} \alpha$ star at the tip and a faint tail pointing back towards GL 961. It is clearly detected in $\mathrm{H} \alpha$, but it is not detected in [NII]. There appears to be a very faint sheath of molecular hydrogen emission corresponding to feature B in the map of
Aspin (1998). However, it is not the optical counterpart of any of the brighter molecular hydrogen features, which may due to orientation and extinction variations. As with the molecular hydrogen bow shocks, it is not possible to uniquely identify the powering source of the optical bow shock, but GL 961-W seems to be the more likely driving source. None of the features detected in $\mathrm{H} \alpha$ were seen in either of the [SII] lines.

\subsection{GL 989 (NGC 2264 IRS1)}

GL 989 is an infrared source discovered by Allen (1972), which is located at a distance of $750 \mathrm{pc}$ and has a luminosity of $4 \times 10^{3} L_{\odot}$. The YSO is at the apex of an extended monopolar nebula at $\mathrm{PA} \sim 315^{\circ}$ that can be seen both in the optical (Allen 1972; Scarrott \& Warren-Smith 1989) and in the nearIR (Schreyer et al. 1997). The source has some intermediate velocity $\mathrm{CO}$ emission, but despite the monopolar nebula little evidence of an ordered bipolar CO flow has been seen (Kruegel et al. 1987). Schreyer et al. (1997) studied the CS 5-4 transition and found a compact outflow along the N-S direction with the blue lobe towards the south. This is not consistent with the monopolar nebula extending to the NW, which would normally correspond to the blueshifted lobe for a reflection nebula. However, polarisation studies by Scarrott \& Warren-Smith (1989) also called into question a simple reflection scenario for this nebula. Recent mm continuum interferometric observations by (Nakano et al. 2003) failed to detect any dense cool dust around the source, which may point to a more evolved status than other MYSOs.

GL 989 was observed with the TTF at one spatial position, to the NW of the IR source, with longest FOV dimension oriented along the nebular direction. Both settings, $\mathrm{H} \alpha$ and [SII] were used. Figure ?? shows the $\mathrm{H} \alpha$ frame (before continuum subtraction) for this region. At the bottom left of the image the optical nebula studied by Scarrott \& Warren-Smith (1989) can be seen, with the IR point source at its apex. The $\mathrm{H} \alpha$ image, after continuum subtraction, is shown in Fig. ??. We do not show the [NII] continuum-subtracted image, since no emission feature was found in the field. It should be noted that the atmospheric conditions were very poor during the observations of this object, which yielded a poor continuum subtraction. In addition, there is an etalon drift of $10 \AA$ between the pencil-beam scans taken before and after observing this source. Therefore, the quality of the line images is the worst in the sample, and the fluxes are affected by higher systematic errors. No significant emission associated with GL 989 is detected in the continuum-subtracted [SII] images. An upper limit of $7 \times 10^{-6} \mathrm{erg} \mathrm{s}^{-1} \mathrm{~cm}^{-2} \mathrm{sr}^{-1}$ is estimated for the [SII] 6717 and 6731 A lines, near the IR source.

The optical nebulosity associated with GL 989 appears to emit in $\mathrm{H} \alpha$. The apparent main orientation of the $\mathrm{H} \alpha$ emission forms an angle of $\sim 30^{\circ}$ with respect to the main direction of emission in $K$ band. A similar orientation was found by Schreyer et al. (1997).

The $\mathrm{H} \alpha$ and the [SII] $6717 \AA$ images also show several extended features distributed inhomogeneously over the whole field of view (see Figs. ?? and ??). This emission is part of 
the extended NGC 2264 nebula. Some of these features have the appearance of filaments. Three of them, which show a bow shape indicating their shocked nature, correspond with the HH objects HH 579, HH 580 and HH 583, detected by Reipurth et al. (2004b). The orientation of the bow shock located at the centre of the field (HH 580), clearly discards GL 989 as the possible driving source. This is most likely associated with the molecular outflow NGC 2264 D (Margulis et al. 1988). HH 579, situated to the NE of GL 989, which is most clearly seen at the bottom left of the $\mathrm{H} \alpha$ frame (Fig. ??) appears at the tail of a bright filamentary emission feature east of GL 989. This bow shock does point back towards GL 989, but does not align with the outflow orientation implied by the optical nebula in GL 989 or the N-S flow in CS (Schreyer et al. 1997).

The objects HH580 and HH 583 show up clearly in the [SII] 6717 transition, $\sim 2^{\prime}$ towards the N-NE of GL 989. In particular, we see that HH 580 is formed by three compact knots (see also Reipurth et al. 2004b). These knots are barely detected in the $\mathrm{H} \alpha$ line as well as in the [SII] 6731 line (the latter not shown in this paper). The fact that they are brighter in [SII] indicates that they are likely of shock origin. These knots do not appear to be associated with GL 989.

\section{Line ratios and strengths}

To further investigate the nature of the features detected with the TTF observations as well as the upper limits, we compare the line ratios and strengths with other observations and radiative shock models available in the literature.

Figure 15 shows the diagnostic ratio of fluxes [NII] $6583 / \mathrm{H} \alpha$ against $[\mathrm{SII}] / \mathrm{H} \alpha$. The error bars for the TTF data are the result of the propagation of the statistical errors listed in Table 2. The systematic errors are canceled out for the $[\mathrm{NII}] / \mathrm{H} \alpha$ ratio since both lines where measured in the same wavelength setting, but not for the [SII]6731/H $\alpha$ ratio. This diagram has the advantage that it is hardly affected by extinction.

Our data are compared to data from a sample of $\mathrm{HH}$ objects associated with low mass YSOs (Dopita 1978) and from a sample of photoionised HII regions (Hunter 1992). Also shown are a series of radiative shock models from Dopita \& Sutherland (1996). Reddening needs to be taken into account when comparing the models to these data. The key diagnostic between a shocked or photoionised origin is the $[\mathrm{SII}] / \mathrm{H} \alpha$ ratio, which is much higher in shocked regions. Dopita \& Sutherland (1995) and Dopita \& Sutherland (1996) indicate that for shocks in the range from $150 \mathrm{~km} \mathrm{~s}^{-1}$ to $500 \mathrm{~km} \mathrm{~s}^{-1}$ the $[\mathrm{SII}] / \mathrm{H} \alpha$ ratio varies between 0.2 (slow shocks) and 1.6 (fast shocks), while for HII regions it is tipically $<0.3$. Unfortunately, we only have upper limits on the [SII] line for the features detected in $\mathrm{H} \alpha$. Although, these are close to the border between the shocked and photoionised regions of the diagram, the uncertainties due to systematic errors and reddening mean that a shock origin cannot be ruled out with confidence for any of the features.

We now look at what constraints the absolute strengths and upper limits on the $\mathrm{H} \alpha$ line put on shock scenarios. The typical speeds in the few jets seen so far from luminous YSOs are of the order of $500 \mathrm{~km} \mathrm{~s}^{-1}$. This is similar to the lower limits

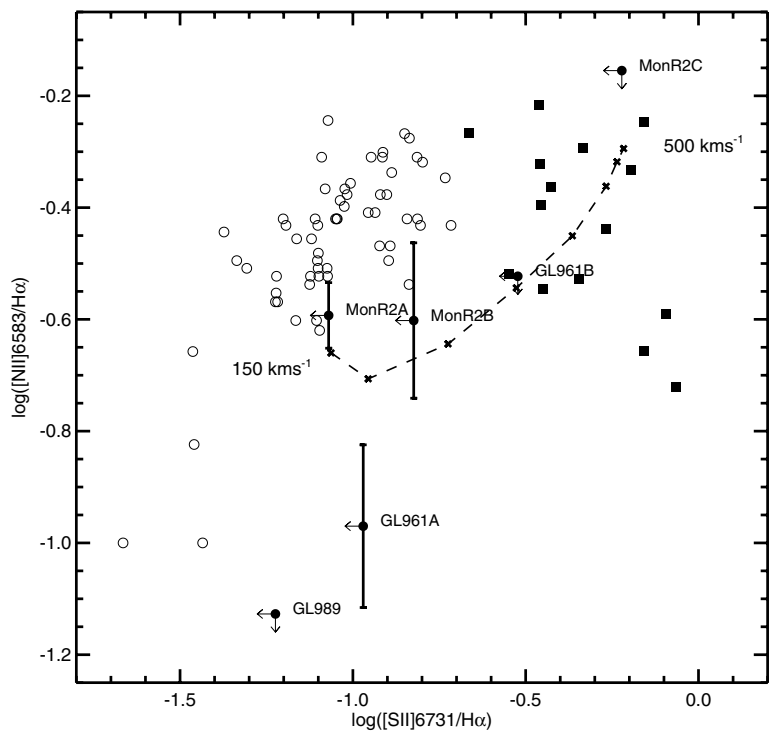

Fig. 15. The observed flux ratios from the sources studied with the TTF (solid circles) are compared with HH objects associated with low mass YSOs from Dopita (1978) (filled squares), photoionised HII regions from Hunter (1992) (open circles) and radiative shock models from Dopita et al. (1996) for for a number density of $1 \mathrm{~cm}^{-3}$ and no magnetic field. The crosses along the lines indicate the shock velocity in increasing value from the bottom to the top of the plot. They range between 150 and $500 \mathrm{~km} \mathrm{~s}^{-1}$ at intervals of $50 \mathrm{~km} \mathrm{~s}^{-1}$.

for the velocity of the ionised winds of a few $100 \mathrm{~km} \mathrm{~s}^{-1}$ that can be inferred from the FWZIs of the HI emission line profiles in massive YSOs (Bunn et al. 1995). The shock models predict an $\mathrm{H} \alpha$ surface brightness of $\sim 10^{-2} \mathrm{erg} \mathrm{s}^{-1} \mathrm{~cm}^{-2} \mathrm{sr}^{-1}$ for a shock velocity of $\sim 500 \mathrm{~km} \mathrm{~s}^{-1}$. Hence, a visual extinction of at least 10 mag would be necessary to reduce this flux below the detection limit of our TTF observations.

To estimate the extinction in the outer parts of the clouds we have inspected optically thin CO isotopomer maps in the literature which are a good measure of column density. (Tafalla et al. 1997) observed ${ }^{13}$ CO $2-1$ line in Mon R2. The integrated line strength at a distance of $1-3^{\prime}$ from the IRS 3 in the SSW direction of the near-IR reflection nebula is about $30-70 \mathrm{~K} \mathrm{~km} \mathrm{~s}^{-1}$. They calculate that this corresponds to a hydrogen column density of $1.0-2.3 \times 10^{22}$ where we have adopted the usual ${ }^{13} \mathrm{CO} / \mathrm{H}$ ratio of $2 \times 10^{-6}$. Using the gas to reddening value from (Bohlin et al. 1978) and a standard extinction law we arrive at a visual extinction of 6-14 mag. Similarly Heyer et al. (1989) find hydrogen column densities of $0.8-1.3 \times 10^{22}$ along the WSW outflow direction in S255 using the ${ }^{13} \mathrm{CO} 1-0$ line corresponding to $A_{\mathrm{V}}$ of 5-7. In GL961 the IRAM ${ }^{13} \mathrm{CO} 2-1$ map shows that the extinction drops to about 3 mag $1^{\prime}$ away from the core. GL989 is unusual here in that there is negligible ${ }^{13} \mathrm{CO}$ emission in the NW direction of the optical nebula, although of course this is consistent with actually seeing the nebulosity.

The visual extinctions in the other sources are sufficiently high that we expect the molecular component to dominate and hence a good measure of the column density. Conversely it is not so high that the ${ }^{13} \mathrm{CO}$ lines become optically thick, as illustrated by $\mathrm{C}^{18} \mathrm{O}$ observations by Ridge et al. (2003), although 


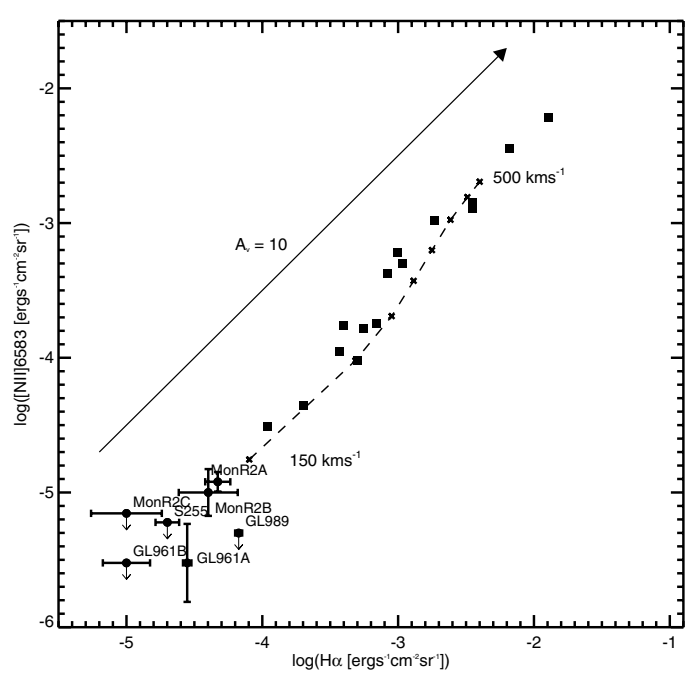

Fig. 16. The observed fluxes from the TTF features (solid circles) are compared with the de-reddened fluxes from the $\mathrm{HH}$ objects studied by Dopita (1978) (filled squares) and with the predictions from radiative shock models. The velocity values are the same as in Fig. 15 and they appear in increasing order from the bottom to the top of the plot.

that is often the case in the core. So at the distances from the sources where we have been searching for shock interactions we find typical visual extinctions of 5-10 mag. Of course, this is through the whole cloud and on average we would expect only about half of this again to any shocked region inside the cloud.

Therefore, on the basis of these values, if shocks of $500 \mathrm{~km} \mathrm{~s}^{-1}$ were present we would have detected them. Any flows would have to be considerably slower or interact obliquely with the surroundings to avoid detection in these optical lines. If we assume that the extinction is only $A_{v}=5$, the model grid by Dopita \& Sutherland (1995) predicts an $\mathrm{H} \alpha$ intensity of $\sim 7 \times 10^{-6} \mathrm{erg} \mathrm{s}^{-1} \mathrm{~cm}^{-2} \mathrm{sr}^{-1}$ for a shock of $150 \mathrm{~km} \mathrm{~s}^{-1}$, which is just above our detection limit. Hence, any shock slower than $150 \mathrm{~km} \mathrm{~s}^{-1}$ would not have been detected in our images. A jet with a speed of $500 \mathrm{~km} \mathrm{~s}^{-1}$ interacting obliquely in a shock at an angle of $\sim 75^{\circ}$ (with respect to the normal to the shock) or larger, will be equivalent to a head-on shock at a speed $<150 \mathrm{~km} \mathrm{~s}^{-1}$, which would therefore be undetected.

One caveat here is that the $\mathrm{CO}$ measurements used to estimate the extinctions are single-dish and fairly large beams. Considerable cloud structure is likely at smaller scales which could lead to locally much higher obscuration. This could well be exactly where the shocked regions are in any scenario where material gets swept up ahead of the outflow. Such action would naturally shield the interaction zone in a dense shell which could well forego any optical emission lines escaping the region. Only higher resolution observations can illuminate this question.

\section{H $\alpha$ emission line stars}

We found in all of the regions several $\mathrm{H} \alpha$ emission line stars. In addition, we also found several stars which show absorption in
$\mathrm{H} \alpha$. In Table 3, we list the stars with the most prominent emission and absorption features, and also give an estimate of the $\mathrm{H} \alpha$ equivalent width $(E W)$. Other stars that also appear to have $\mathrm{H} \alpha$ emission in some of our images are not listed in Table 3 because the error in their $E W$ was too large, and some of them also show up in emission in the [NII] lines. The $\mathrm{H} \alpha E W$ s were calculated from the ratio between the flux measured in the line images and the continuum images, by using aperture photometry. The errors quoted correspond to the propagation through the ratio of the errors in the photometry. Since the emission line stars are in massive star forming regions, we suspect that these are Herbig $\mathrm{Ae} / \mathrm{Be}$ stars. Their $\mathrm{H} \alpha E W \mathrm{~s}$ are consistent with those typically found in the surveys by Finkenzeller \& Mundt (1984) and Herbig \& Bell (1988). The absorption line stars have EWs consistent with normal late B stars (Buscombe 1969).

We have searched in the SIMBAD database for possible cross-identifications of these stars, which are listed in the last column of Table 3. One of them (Mon R2 N 2) is a known X-ray source. This is consistent with this star having also $\mathrm{H} \alpha$ emission, since X-ray emission is thought to be associated with accretion in young stellar objects, although it can also be linked to magnetospheric effects.

In the S255 region, three stars show clear $\mathrm{H} \alpha$ absorption (see Table 3). For the other stars that show apparent absorption in Fig. ??b, EWs are not given in Table 3 because of the strong errors due to their location at the edges of the bright optical HII regions S255 and S257. In the GL 961 region, we found two emission line stars, one of them (star 2) coincident with an near-IR star.

In the region around GL 989, we clearly detect $13 \mathrm{H} \alpha$ emission line stars. All but 2 were also identified in the recent survey by Reipurth et al. (2004a). Many of our detections are previously known as X-ray sources. Reipurth et al. (2004a) identified 13 more stars in the area covered by our field. About 8 of these were classified as weak $\mathrm{H} \alpha$ emitters by Reipurth et al. (2004a). These appear to be also very weak in our $\mathrm{H} \alpha$ image, and were not listed in Table 3, since their EWs were not reliable. The remaining 5 were classified as strong $\mathrm{H} \alpha$ emitters by Reipurth et al. (2004a). The $E W$ s have large errors in 4 of them, probably due to the presence of bright nearby stars, and 1 is very weak in our $\mathrm{H} \alpha$ image.

We have compared our measured $E W \mathrm{~s}$ in three of the previously known bright emission line stars $(\operatorname{LkH} \alpha 35, \mathrm{LkH} \alpha 43$, $\mathrm{LkH} \alpha 47)$ with measurements found in Herbig \& Bell (1988). The larger discrepancy between our $\mathrm{H} \alpha E W$ and the literature value is factor of 3 for $\mathrm{LkH} \alpha 47$. This can be explained due to the strong variability that emission line stars are known to manifest. Finkenzeller \& Mundt (1984), in a comparison between the $E W$ 's that they measured and previous measurements by Garrison \& Anderson (1977), found discrepancies as large as a factor of 2.7 (for $\mathrm{Lk} \mathrm{H} \alpha 234$ ). Typical discrepancies in $E W$ 's by a factor of $\sim 2$ were found in several other sources. Furthermore, they found highly variable $\mathrm{H} \alpha$ profiles, which could affect the $E W$ 's due to large shifts in the peak position. They found, for instance, a shift of $\sim 250 \mathrm{~km} \mathrm{~s}^{-1}$ in the $\mathrm{H} \alpha$ peak (i.e. $5.5 \AA$ ) of HD 53367, in a timescale of 6 months. Given the narrow bandpass of our TTF observations, such shifts may affect our values of the $E W$ 's. Other indications of variability in emission line 
Table 3. Emission line stars ${ }^{a}$.

\begin{tabular}{|c|c|c|c|c|c|c|c|}
\hline Region & ID & $\alpha(\mathrm{hms})$ & $\delta\left(^{(\prime \prime \prime}\right)$ & $E W(\mathrm{H} \alpha)^{a}$ & Refs. ${ }^{b}$ & Notes $^{c}$ & Names $^{d}$ \\
\hline \multirow[t]{3}{*}{ Mon R2 N } & 3 & 060746.2 & -062026 & $-24 \pm 3$ & - & 1 & - \\
\hline & 2 & 060752.2 & -062117 & $-31 \pm 2$ & 1,2 & 1,2 & [CMD97] 380 \\
\hline & 1 & 060753.5 & -061726 & $-35 \pm 3$ & 1 & 1 & [CMD97] 1348 \\
\hline \multirow[t]{2}{*}{ Mon R2 S } & 1 & 060751.6 & -062601 & $-52 \pm 4$ & 1 & 1 & [CMD97] 1322 \\
\hline & 2 & 060751.7 & -062628 & $-114 \pm 60$ & 1 & 1 & [CMD97] 1325 \\
\hline \multirow[t]{8}{*}{ S255 } & 2 & 061236.5 & 175627 & $0.9 \pm 0.1$ & - & 4 & - \\
\hline & 5 & 061240.8 & 175611 & $-3.7 \pm 0.8$ & - & 1 & - \\
\hline & 3 & 061240.8 & 175835 & $-20 \pm 4$ & - & 1 & - \\
\hline & 4 & 061241.0 & 175945 & $13 \pm 2$ & - & 4 & - \\
\hline & $8^{*}$ & 061247.3 & 180013 & - & - & 4 & - \\
\hline & 1 & 061251.3 & 175913 & $1.3 \pm 0.2$ & - & 4 & - \\
\hline & $7^{*}$ & 061257.6 & 175823 & - & - & 4 & - \\
\hline & $6^{*}$ & 061259.5 & 175722 & - & - & 4 & - \\
\hline \multirow[t]{2}{*}{ GL 961} & $1^{* *}$ & 063435.7 & 041245 & $-22 \pm 8$ & - & 1 & - \\
\hline & $2^{* *}$ & 063437.9 & 041313 & $-42 \pm 9$ & - & 1 & - \\
\hline \multirow[t]{13}{*}{ GL 989} & 7 & 064047.1 & 093240 & $-9 \pm 1$ & $3,5,6,7,8,10$ & 2,3 & $\mathrm{LkH} \alpha 26$ \\
\hline & 6 & 064053.6 & 093326 & $-12 \pm 2$ & $3,5,6,7,8,10$ & 2,3 & $\mathrm{LkH} \alpha 35$ \\
\hline & 9 & 064056.7 & 093018 & $-5.9 \pm 0.6$ & $3,6,7,8$ & 2,3 & NGC 2264 FMS 281 \\
\hline & 8 & 064058.8 & 093059 & $-11 \pm 1$ & $5,6,7,8,9,10$ & 3 & $\mathrm{LkH} \alpha 43$ \\
\hline & 10 & 064059.4 & 092953 & $-6.2 \pm 0.6$ & $4,6,7,10$ & 3 & KHA 105 \\
\hline & 4 & 064101.0 & 093245 & $-4.2 \pm 0.4$ & $3,5,6,7,8,10$ & 2,3 & $\mathrm{LkH} \alpha 47$ \\
\hline & 5 & 064101.3 & 093409 & $-4.5 \pm 0.6$ & $3,6,7,8$ & 1,2 & NGC 2264 FMS 315 \\
\hline & 12 & 064103.5 & 093029 & $-10 \pm 1$ & 7,10 & 3 & $\mathrm{ESOH} \alpha 473$ \\
\hline & 3 & 064105.7 & 093101 & $-3.1 \pm 0.2$ & 7,10 & 3 & $\mathrm{ESOH} \alpha 486$ \\
\hline & 13 & 064106.9 & 092925 & $-16 \pm 2$ & 7,10 & 3 & $\mathrm{ESOH} \alpha 492$ \\
\hline & 1 & 064109.1 & 093010 & $-51 \pm 9$ & $3,5,6,7,8,10$ & 2,3 & $\mathrm{LkH} \alpha 58$ \\
\hline & 2 & 064108.2 & 093023 & $-84 \pm 16$ & 6,10 & 3 & $\mathrm{ESOH} \alpha 497$ \\
\hline & 11 & 064108.9 & 093112 & $-28 \pm 3$ & 10 & 3 & $\mathrm{ESOH} \alpha 501$ \\
\hline
\end{tabular}

${ }^{a}$ Equivalent widths in $\AA$. A negative sign represents emission and a positive sign absorption.

$b$ References: (1) Carpenter et al. (1997); (2) Kohno et al. (2002); (3) Flaccomio et al. (2000); (4) Ogura (1984); (5) Herbig (1954); (6) Flaccomio et al. (1999); (7) Rebull et al. (2002); (8) Sung et al. (1997); (9) Herbig \& Bell (1988); (10) Reipurth et al. (2004a).

c Notes: (1) unknown $\mathrm{H} \alpha$ star; (2) X-ray source; (3) known $\mathrm{H} \alpha$ source; (4) $\mathrm{H} \alpha$ in absorption.

$d$ Known names.

* No $E W$ is given because of the large errors due to the location of the star at the edge of the extended HII regions S255 and S257.

** Coincide in position with the the extended features A and B. Star 2 corresponds with a near-IR star (see Sect. 3).

stars have been obtained in spectropolarimetric studies (e.g. Oudmaijer \& Drew 1999).

\section{Conclusions}

The TTF was used to search for optical shock-excited emission from the regions where we expect outflows to interact with interstellar matter in four massive YSOs. No clear emission was found that can be related with the molecular outflow, except possibly for the feature B in Mon R2. In the same region, feature A appears to be consistent with being part of the HII region excited by IRS1. A similar case appears to be the emission seen in S255 IRS2. This is confirmed by the limits on the line ratios.
The ionised flows that emanate from massive YSOs have speeds of order $500 \mathrm{~km} \mathrm{~s}^{-1}$. If these flows were colliding headon with the ISM then we require extinctions of at least $A_{\mathrm{v}}=10$ to explain the non-detection of shock-excited emission we have observed. Extinctions this high towards the interaction zones cannot be ruled out, although reasonable estimates are somewhat lower than this. If the extinction is low, then it implies that such shock interactions are not present in these massive outflows, and that the kind of jet-driven bow shocks common in low-mass outflows are not the usual driving mechanism for high-mass outflows. In this case our data would be more consistent with a picture in which any fast flows from massive YSOs have a wide opening angle, and therefore shock obliquely when 
interacting with the ISM or have been significantly slowed before any head-on interaction.

Before more definitive statements can be made on the outflow driving and interaction, higher resolution millimetre studies of the outflow and molecular cloud need to be made. These would locate precisely where the outflow terminates and pinpoint where it appears that momentum is being transfer into the gas by a shock. With suitable line or dust continuum tracers much better estimates of the column density and extinction to the emission regions could be made. This could then guide future deep searches for shocked optical emission with IFUs on large telescopes.

Deeper searches in the near-IR, where the extinction is lower, are also required. If wide-angled or equatorial winds are the norm in massive YSOs, we should be able to trace their working surfaces in slow shocks or at oblique angles using near-IR $\mathrm{H}_{2}$ imaging. These working surfaces maybe very close to the driving source. In such cases high resolution techniques (e.g. Adaptive Optics) will be necessary. Future high resolution radio mapping, e.g. with e-MERLIN, will be able to elucidate the geometry of the ionised winds very close to the driving source. This will also point the way to where we should search for the interaction of these flows with the ISM.

Acknowledgements. C.A. would like to thank to the School of Physics and Astronomy at Leeds University for their support. C.A. is also deeply grateful to Kapteyn Astronomical Institute for allowing him to use their facilities during the realization of this work. We thank the anonymous referee for the useful comments and suggestions which have helped to improve the quality of this manuscript. This research has made use of the SIMBAD database, operated at CDS, Strasbourg, France.

\section{References}

Allen, D. A. 1972, ApJ, 12, L231

Alvarez, C., Hoare, M., Glindemann, A., \& Richichi, A. 2004, A\&A, 427,505

Aspin, C. 1998, A\&A, 335, 1040

Aspin, C., \& Walther, D. M. 1990, A\&A, 235, 387

Bally, J., \& Lada, C. J. 1983, ApJ, 265, 824

Beckwith, S., Evans, N. J. I., Becklin, E. E., \& Neugebauer, G. 1976, ApJ, 208, 390

Beetz, M., Elsaesser, H., Weinberger, R., \& Poulakos, C. 1976, A\&A, 50,41

Beuther, H., Schilke, P., Sridharan, T. K., et al. 2002, A\&A, 383, 892

Bland-Hawthorn, J., \& Jones, D. H. 1998, in Optical Astronomical Instrumentation, ed. S. D'Odorico, Proc. SPIE, 3355, 855

Bohlin, R. C., Savage, B. D., \& Drake, J. F. 1978, ApJ, 224, 132

Bunn, J. C., Hoare, M. G., \& Drew, J. E. 1995, MNRAS, 272, 346

Burrows, C. J., Stapelfeldt, K. R., Watson, A. M., et al. 1996, ApJ, 473, 437

Buscombe, W. 1969, MNRAS, 144, 31

Carpenter, J. M., Meyer, M. R., Dougados, C., Strom, S. E., \& Hillenbrand, L. A. 1997, AJ, 114, 198

Castelaz, M. W., Grasdalen, G. L., Hackwell, J. A., Capps, R. W., \& Thompson, D. 1985, AJ, 90, 1113

Coffey, D., Bacciotti, F., Woitas, J., Ray, T. P., \& Eislöffel, J. 2004, ApJ, 604, 758

Davis, C. J., Moriarty-Schieven, G., Eislöffel, J., Hoare, M. G., \& Ray, T. P. 1998, AJ, 115, 1118
Delamarter, G., Frank, A., \& Hartmann, L. 2000, ApJ, 530, 923

Dopita, M. A. 1978, ApJS, 37, 117

Dopita, M. A., \& Sutherland, R. S. 1995, ApJ, 455, 468

Dopita, M. A., \& Sutherland, R. S. 1996, ApJS, 102, 161

Drew, J. E., \& Proga, D. 2000, New Astron. Rev., 44, 21

Eiroa, C. 1981, A\&AS, 44, 77

Eislöffel, J. 2000, A\&A, 354, 236

Evans, N. J., Blair, G. N., \& Beckwith, S. 1977, ApJ, 217, 449

Finkenzeller, U., \& Mundt, R. 1984, A\&AS, 55, 109

Flaccomio, E., Micela, G., Sciortino, S., et al. 2000, A\&A, 355, 651

Flaccomio, E., Micela, G., Sciortino, S., et al. 1999, A\&A, 345, 521

Garrison, L. M., \& Anderson, C. M. 1977, ApJ, 218, 438

Georgelin, Y. M., Georgelin, Y. P., \& Roux, S. 1973, A\&A, 25, 337

Gibb, A. G., Hoare, M. G., Little, L. T., \& Wright, M. C. H. 2003, MNRAS, 339, 1011

Goetz, J. A., Pipher, J. L., Forrest, W. J., et al. 1998, ApJ, 504, 359

Gunn, J. E., \& Stryker, L. L. 1983, ApJS, 52, 121

He, L., Whittet, D. C. B., Kilkenny, D., \& Spencer Jones, J. H. 1995, ApJS, 101, 335

Henning, T., Schreyer, K., Launhardt, R., \& Burkert, A. 2000, A\&A, 353,211

Herbig, G. H. 1954, ApJ, 119, 483

Herbig, G. H., \& Bell, K. R. 1988, Catalog of emission line stars of the orion population: 3, Lick Observatory Bulletin, Santa Cruz: Lick Observatory

Heyer, M. H., Snell, R. L., Morgan, J., \& Schloerb, F. P. 1989, ApJ, 346,220

Hoare, M. G. 2002, in The Earliest Stages of Massive Star Birth, ed. P. A. Crowther, ASP Conf. Proc., 267, 137

Hoare, M. G., Glindemann, A., \& Richichi, A. 1996, in The Role of Dust in the Formation of Stars, ESO Proc., ed. Käufl, \& Siebenmorgen, 35

Hodapp, K. 1994, ApJS, 94, 615

Howard, E. M., Pipher, J. L., \& Forrest, W. J. 1994, ApJ, 425, 707

Howard, E. M., Pipher, J. L., \& Forrest, W. J. 1997, ApJ, 481, 327

Hunter, D. A. 1992, ApJS, 79, 469

Kohno, M., Koyama, K., \& Hamaguchi, K. 2002, ApJ, 567, 423

Konigl, A. 1982, ApJ, 261, 115

Koresko, C. D., Beckwith, S., Ghez, A. M., et al. 1993, AJ, 105, 1481

Kruegel, E., Guesten, R., Schulz, A., \& Thum, C. 1987, A\&A, 185, 283

Kumar, M. S. N., Bachiller, R., \& Davis, C. J. 2002, ApJ, 576, 313

Lada, C. J., \& Gautier, T. N. I. 1982, ApJ, 261, 161

Lee, C., Stone, J. M., Ostriker, E. C., \& Mundy, L. G. 2001, ApJ, 557, 429

Lenzen, R., Hodapp, K. W., \& Reddmann, T. 1984, A\&A, 137, 365

Margulis, M., Lada, C. J., \& Snell, R. L. 1988, ApJ, 333, 316

Marti, J., Rodriguez, L. F., \& Reipurth, B. 1993, ApJ, 416, 208

Marti, J., Rodriguez, L. F., \& Reipurth, B. 1998, ApJ, 502, 337

Massi, M., Felli, M., \& Simon, M. 1985, A\&A, 152, 387

Masson, C. R., \& Chernin, L. M. 1994, in Clouds, Cores, and Low Mass Stars, ASP Conf. Ser., 65, 350

Miralles, M. P., Salas, L., Cruz-Gonzalez, I., \& Kurtz, S. 1997, ApJ, 488, 749

Mitchell, G. F., Maillard, J.-P., \& Hasegawa, T. I. 1991, ApJ, 371, 342

Mundt, R., \& Ray, T. P. 1994, in The Nature and Evolutionary Status of Herbig Ae/Be Stars, ASP Conf. Ser., 62, 237

Nakano, M., Sugitani, K., \& Morita, K. 2003, PASJ, 55, 1

Ogura, K. 1984, PASJ, 36, 139

Osterbrock, D. E. 1989, Astrophysics of gaseous nebulae and active galactic nuclei (University Science Books), 422

Oudmaijer, R. D., \& Drew, J. E. 1999, MNRAS, 305, 166

Poetzel, R., Mundt, R., \& Ray, T. P. 1992, A\&A, 262, 229 
Preibisch, T., Balega, Y. Y., Schertl, D., \& Weigelt, G. 2002, A\&A, 392, 945

Proga, D., Stone, J. M., \& Drew, J. E. 1998, MNRAS, 295, 595

Ray, T. P., Poetzel, R., Solf, J., \& Mundt, R. 1990, ApJ, 357, L45

Rebull, L. M., Makidon, R. B., Strom, S. E., et al. 2002, AJ, 123, 1528

Reipurth, B., Bally, J., \& Devine, D. 1997, AJ, 114, 2708

Reipurth, B., Pettersson, B., Armond, T., Bally, J., \& Vaz, L. P. R. 2004a, AJ, 127, 1117

Reipurth, B., Yu, K., Moriarty-Schieven, G., et al. 2004b, AJ, 127, 1069

Ridge, N. A., \& Moore, T. J. T. 2001, A\&A, 378, 495

Ridge, N. A., Wilson, T. L., Megeath, S. T., Allen, L. E., \& Myers, P. C. 2003, AJ, 126, 286

Rodríguez, L. F., Torrelles, J. M., Anglada, G., \& Martí, J. 2001, Rev. Mex. Astron. Astrofis., 37, 95

Scarrott, S. M., \& Warren-Smith, R. F. 1989, MNRAS, 237, 995

Schreyer, K., Helmich, F. P., van Dishoeck, E. F., \& Henning, T. 1997, A\&A, 326, 347
Shepherd, D. S., Watson, A. M., Sargent, A. I., \& Churchwell, E. 1998, ApJ, 507, 861

Shu, F., Najita, J., Ostriker, E., et al. 1994, ApJ, 429, 781

Snell, R. L., \& Bally, J. 1986, ApJ, 303, 683

Sung, H., Bessell, M. S., \& Lee, S. 1997, AJ, 114, 2644

Tafalla, M., Bachiller, R., Wright, M. C. H., \& Welch, W. J. 1997, ApJ, 474, 329

Tamura, M., Gatley, I., Joyce, R. R., et al. 1991, ApJ, 378, 611

Thronson, H. A., Gatley, I., Harvey, P. M., Sellgren, K., \& Werner, M. W. 1980, ApJ, 237, 66

Tofani, G., Felli, M., Taylor, G. B., \& Hunter, T. R. 1995, A\&AS, 112, 299

Torrelles, J. M., Gomez, J. F., Rodriguez, L. F., et al. 1996, ApJ, 457, L107

Wang, H., Yang, J., Wang, M., \& Yan, J. 2003, AJ, 125, 842

Wolf, G. A., Lada, C. J., \& Bally, J. 1990, AJ, 100, 1892

Yao, Y., Hirata, N., Ishii, M., et al. 1997, ApJ, 490, 281 